\title{
Plasmacytoid dendritic cell-derived IFN $\alpha$ modulates Th17 differentiation during early Bordetella pertussis infection in mice
}

\author{
V Wu ${ }^{1}$, AA Smith ${ }^{1,2}$, H You $^{1,2}$, TA Nguyen ${ }^{1,2,3}$, R Ferguson ${ }^{1}$, M Taylor $^{1}$, JE Park ${ }^{1}$, P Llontop ${ }^{1}$, \\ KR Youngman ${ }^{1}$ and $\mathrm{T}$ Abramson ${ }^{1}$
}

Whooping cough is a highly contagious respiratory disease caused by Bordetella pertussis (B. pertussis). T helper 17 (Th17) cells have a central role in the resolution of the infection. Emerging studies document that type I interferons (IFNs) suppress Th17 differentiation and interleukin (IL)-17 responses in models of infection and chronic inflammation. As plasmacytoid dendritic cells ( $\mathrm{pDCs}$ ) are a major source of type I IFNs, we hypothesize that during B. pertussis infection in mice, pDC-derived IFN $\alpha$ inhibits a rapid increase in Th17 cells. We found that IFN $\alpha$-secreting pDCs appear in the lungs during the early stages of infection, while a robust rise of Th17 cells in the lungs is detected at 15 days post-infection or later. The presence of IFN $\alpha$ led to reduced Th17 differentiation and proliferation in vitro. Furthermore, in vivo blocking of IFN $\alpha$ produced by pDCs during infection with $B$. pertussis infection resulted in early increase of Th17 frequency, inflammation, and reduced bacterial loads in the airways of infected mice. Taken together, the experiments reported here describe an inhibitory role for pDCs and pDC-derived IFN $\alpha$ in modulating Th17 responses during the early stages of $B$. pertussis infection, which may explain the prolonged nature of whooping cough.

\section{INTRODUCTION}

Bordetella pertussis (B. pertussis) is the Gram-negative, extracellular bacterial pathogen that causes whooping cough, a reemerging highly contagious respiratory disease. In both mice and baboons, $\mathrm{T}$ helper 17 (Th17) cells have been documented as crucial for the resolution of this infection. ${ }^{1}$ Baboons infected with $B$. pertussis produced Th17-promoting cytokines (interleukin (IL)-6, IL-23, and IL-1 $\beta$ ) and IL-17 responses in the nasopharynx. ${ }^{2}$ In these studies, IL-17-secreting $\mathrm{CD}^{+}{ }^{+}$cells were detected long after the convalescent period, suggesting a role for Th17 cells in recovery as well as in memory responses. $^{3}$

Several B. pertussis virulence factors, such as adenylate cyclase, lipopolysaccharide, $\mathrm{p} .69$ pertactin, and pertussis toxin (PTX) have been shown to promote IL-17 responses during infection. ${ }^{4-9}$ For example, PTX induction of Th17 responses in mice infected with $B$. pertussis, leading to subsequent neutrophil recruitment and clearance of infection, has been well described. ${ }^{10,11}$ IL-17 also enhances cytokine-mediated macrophage killing in vitro. ${ }^{12}$ Conversely, filamentous hemagglutinin suppresses early IL-17 responses and Th17 inflammation in mice infected with $B$. bronchiseptica, a related strain with interchangeable $f h a B$ genes derived from $B$. pertussis. ${ }^{13}$

IL-17 responses, owing to the activation of innate and Th17 cells, have been shown to be critical for the resolution of other mucosal infections as well as the perpetuation of chronic inflammation. ${ }^{14}$ Type I interferons (IFN $\alpha$ and IFN $\beta$ ) display pleiotropic immunomodulatory functions in both conditions. ${ }^{15}$ These cytokines are secreted by diverse types of cells, but primarily by plasmacytoid dendritic cells (pDCs), in response to a variety of TLR7- and TLR9-activating pathogenic molecules. ${ }^{15,16}$ Although type I IFN responses were initially associated with viral or intracellular pathogens, recent studies have demonstrated that extracellular respiratory pathogens can also trigger such responses. Type I IFN responses may be either beneficial or detrimental for the host in each of these

\footnotetext{
${ }^{1}$ Department of Biology, San José State University, San José, California, USA. Correspondence: T Abramson (tzvia.abramson@sjsu.edu)

${ }^{2}$ These authors contributed equally to this work.

${ }^{3}$ Present address: BD Biosciences, 2350 Qume Drive, San José, California 95131, USA. 
a

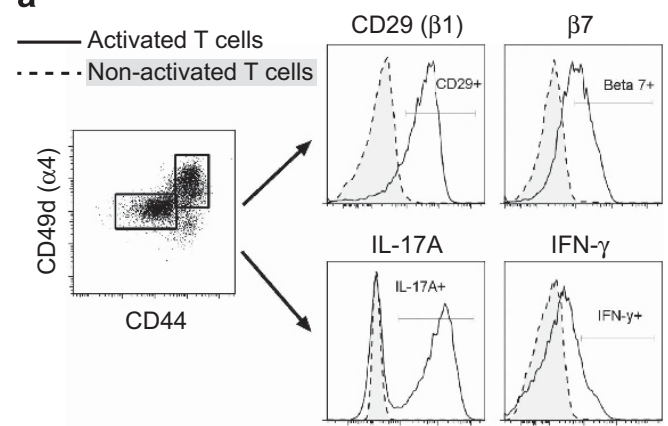

b

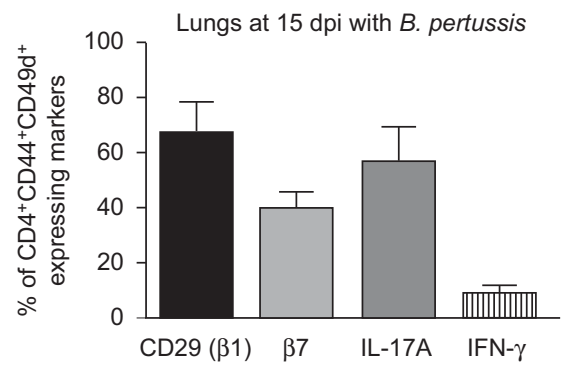

Figure 1 CD49d mucosal trafficking receptor expressing emTh cells at $15 \mathrm{dpi}$ with $B$. pertussis. Mononuclear cells were collected from lungs of mice infected with $B$. pertussis and $\mathrm{CD} 4{ }^{+}$cells were analyzed by flow cytometry and gated on CD44 ${ }^{+}$and CD49d ${ }^{+}$cells. The frequency of cells expressing CD29 and $\beta 7$ was analyzed on extracellular stained cells and IL-17A- and IFN $\gamma$-expressing cells were identified by intracellular staining as detailed in materials and methods. (a) Representative histograms are shown expressing CD29, beta7 and IL-17, and IFN $\gamma$. (b) Summary of expression levels of CD29, beta7 and IL-17, and IFN $\gamma$ by CD4 ${ }^{+} \mathrm{CD} 44^{+}$ CD49d ${ }^{+}$lung cells. (Histograms represent one experiment from three independent experiments. Bar graphs represent average of three independent experiments.) dpi, days post-infection; IFN, interferon; IL, interleukin; Th cells, T helper cells.

pathogenic conditions. ${ }^{17,18}$ For example, in Fracisella tularensis and Listeria monocytogenes infections, IL-17 produced by $\gamma \delta$ $\mathrm{T}$ cells was limited by type I IFN signaling. In these studies, mice deficient in IFN receptors displayed increased survival rates, demonstrating the constricting effects of type I IFN on the resolution of bacterial infection. ${ }^{18}$ At the same time, type I IFN has been shown to be beneficial in alleviating Th17-associated inflammation in ulcerative colitis and encephalomyelitis in mice by promoting IL-10-secreting $\mathrm{T}$ cells and suppressing inflammation. ${ }^{19-21}$ Although the effect of type I IFN during infection with $B$. pertussis is understudied, microarray analysis of human peripheral blood mononuclear cells treated with filamentous hemagglutinin showed a notable upregulation of IFN-regulated genes, suggesting a role for these cytokines in B. pertussis pathogenesis. ${ }^{22}$

Here, we test the hypothesis that pDCs, owing to their secretion of type I IFN, inhibits Th17 responses, thus contributing to early pathogenesis during $B$. pertussis infection. The results documented here suggest an inhibitory role for the early-secreted IFN $\alpha$ on specific responses to $B$. pertussis infection, as well as facilitating early pulmonary colonization with $B$. pertussis in mice.

\section{RESULTS}

\section{Robust rise in IL-17-secreting cells occurs late post-} infection with $B$. pertussis

We previously reported that effector memory $\mathrm{T}$ cells $\left(\mathrm{CD} 4{ }^{+} \mathrm{CD}_{4}{ }^{+} \mathrm{CD} 4 \mathrm{RB}^{-}\right)$expressing mucosal trafficking receptors such as $\alpha 4 \beta 7$ and $\alpha 4 \beta 1$ were significantly elevated in the lungs of mice 15 days after $B$. pertussis infection. ${ }^{23}$ In our current study, we noted that CD $44^{+} \mathrm{CD} 49 \mathrm{~d}^{+}$cells in the infected lungs expressed a mucosal phenotype, as reflected by the combined expression of CD49d ( $\alpha 4)$ with either CD29 or $\beta 7$. About $50 \%$ of these cells expressed IL-17, but less than $10 \%$ expressed IFN $\gamma$, suggesting that activated Th cells migrating to mucosal tissue manifest Th17 polarization (Figure 1a and $\mathbf{b}$ ).

The importance of both Th1 and Th17 cells during the resolution of $B$. pertussis infections was previously reported, and hence, we followed these two effector memory cell types throughout the course of infection. ${ }^{2,3}$ Comparable frequencies of Th1 cells were detected up to 50 days post-infection (dpi), suggesting a supporting role for IFN $\gamma$ and these cells during infection. However, a rise in Th17 cells was not detected until 15 dpi in both the blood and the lungs (Figure 2a-c). A strong presence of these cells was still detected in the lungs at 35 and 50 dpi (Figure 2c). Although IL-17 mRNA expression in lung tissue was upregulated at 5 dpi compared with uninfected tissue, it significantly further augmented at $15 \mathrm{dpi}(P<0.01)$ (Figure 2d). This suggests that increased IL-17 is initially contributed by early immune responses, but the robust increase of IL-17 expression observed in the lungs at 15dpi is in parallel with the rise of pulmonary Th17 cells. Furthermore, histological studies revealed increased inflammation at 15 dpi and later (Figure 2e) ${ }^{23}$ These data suggest that Th17 cells are long lasting, provide an IL-17-rich environment, and sustain inflammation in the lungs for a prolonged period of time.

To verify that the increase in Th17 cells was associated with PTX, as previously reported, we compared the frequency of Th17 cells in mice infected with wild-type $B$. pertussis or the PTX-deficient strain $(B p \Delta P T X) .{ }^{10}$ We observed a clear dependency of the Th17 cell emergence on the presence of PTX, as mice infected with Bp $\triangle P T X$ did not display a robust increase of these cells in either blood or lungs (Figure $\mathbf{2} \mathbf{f}$ and $\mathbf{g}$ ). This suggests that Th17 differentiation is primarily induced by PTX, and is, perhaps, responsible for the prolonged lung inflammation during infection with $B$. pertussis.

\section{IFN $\alpha$-secreting pDCs accumulate in the lungs early post- infection with $B$. pertussis}

To evaluate the role of IFN $\alpha$ in the delayed appearance of Th17 responses in mice infected with $B$. pertussis, we measured the frequency of $\mathrm{pDCs}$ - the primary precursor of this cytokine-in the lungs of infected mice.

We characterized pDCs as CD11c ${ }^{\text {int }}, \mathrm{CD}_{11}{ }^{-}$, and mPDCA- ${ }^{+}$. Consistent with other reports, these cells also expressed B220 and SiglecH (data not shown). ${ }^{15}$ In homeostatic conditions, about $2 \%$ of all lung $\mathrm{CD}_{11 \mathrm{c}^{+}}$cells are pDCs 


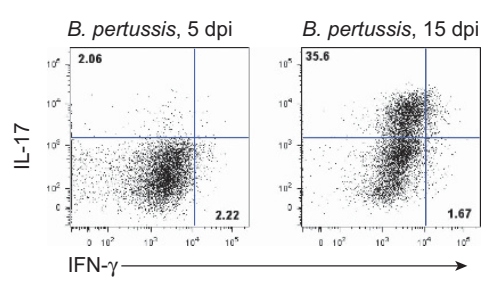

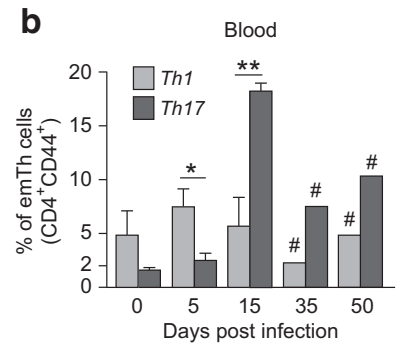

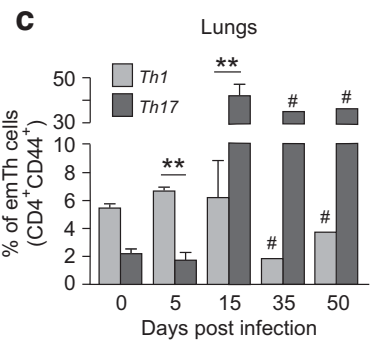

d

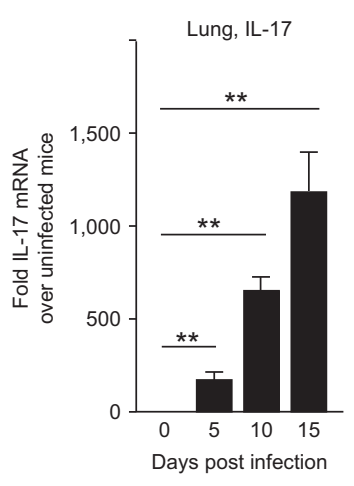

e Uninfected

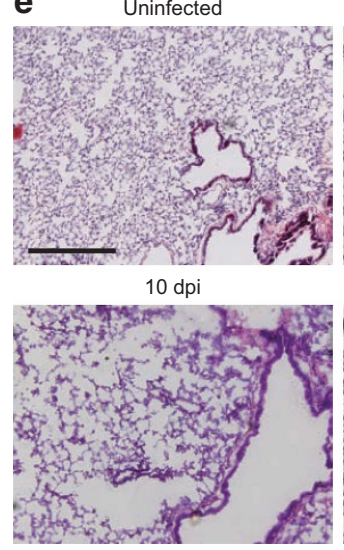

$5 \mathrm{dpi}$

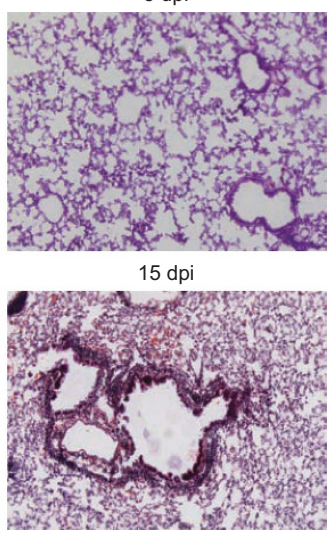

f B. pertussis
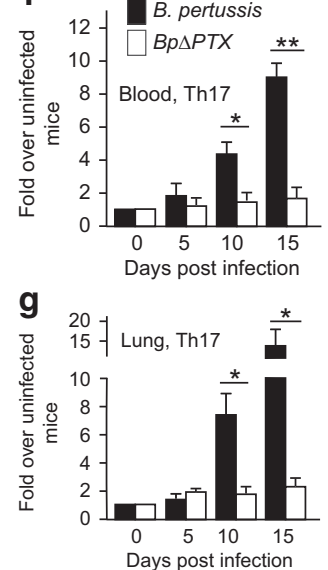

Figure 2 The frequency of Th1/Th17 response throughout $B$. pertussis infection in mice. Mononuclear cells were collected from the blood and lungs of uninfected mice and mice infected with $B$. pertussis as described and were analyzed by flow cytometry and gated on CD4 ${ }^{+}$and CD44 ${ }^{+}$. (a) The frequency of IL-17- or IFN $\gamma$-expressing cells was determined at 5 and $15 \mathrm{dpi}$. (b) The frequency of Th17 cells was compared with Th1 cells at various times post infection in the blood and (c) lungs of infected mice. (d) The relative mRNA expression levels of IL-17 in lung tissue was measured by quantitative RTPCR and normalized to uninfected lung tissue. (e) H\&E staining of lung sections show overall lung inflammation. (f) Cytometric analysis was used to evaluate PTX-associated frequencies of Th17 cells relative to uninfected mice in blood and (g) lungs. Graph expresses fold of CD4 ${ }^{+}$CD $44^{+} \mathrm{IL}-17^{+}$cells over uninfected mice. (Bar graphs represent averages of at least three independent experiments unless otherwise noted; ${ }^{\star} P<0.05$; ${ }^{* \star} P<0.01$; \#, represents one experiment; scale bar $=400 \mu \mathrm{m}$.) dpi, days post-infection; H\&E, hematoxylin and eosin; IFN, interferon; IL, interleukin; PTX, pertussis toxin; Th cells, T helper cells.

(Figure 3a). However, during early post-infection with B. pertussis, the percentage of these cells rises up to about 8\% (Figure 3a). Lung pDCs peak during infection with B. pertussis at $5-10$ dpi before returning to uninfected levels (Figure 3b). Although there is a moderate rise of $\mathrm{pDC}$ in mice

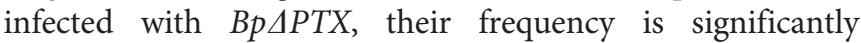
attenuated during the peak of infection compared with mice infected with the wild-type bacteria (Figure $\mathbf{3 b}$ ). This suggests that although PTX is promoting $\mathrm{pDC}$ accumulation in the lungs, it is most likely not the only contributing virulence factor.

Only very small percentages of pDCs were detected in the mediastinal lymph nodes of uninfected mice and at 5 dpi (Figure 3c). However, the frequency of pDCs increased significantly in the mediastinal lymph node starting at 10 dpi (Figure 3d).

$\mathrm{G}$ protein-coupled receptors guide the migration of pDCs into the lymphatics and their entrance to the draining lymph nodes. ${ }^{24-27}$ As predicted, at $10 \mathrm{dpi}$, there are significantly fewer lung pDCs expressing the $\mathrm{G}$ protein-coupled receptors (CCR7 and CXCR4) in lungs of $B$. pertussis-infected mice compared with respective $\mathrm{pDCs}$ derived from $B p \triangle P T X$-infected mice (Figure 3e). This implies a PTX-inhibitory effect on G protein- coupled receptors that may interfere with the ability of pDCs to rapidly exit the lungs into the lymphatics, as evidenced by the high frequency of pDCs that still reside in the lungs at 10 dpi (Figure 3b).

\section{Phenotypic and functional characterization of lung pDCs} As pDCs accumulate rapidly in the lungs during $B$. pertussis infection, we anticipated that these cells would have a significant immunomodulatory contribution in the lung niche. We noted that although the frequency of MHC class II-expressing lung pDCs derived from $B$. pertussis-infected mice is comparable with respective cells from $B p \triangle P T X$ or uninfected mice, CD86 co-stimulatory molecule expression is reduced (Figure 4a and $\mathbf{b}$ ). These data imply that at 5 dpi with B. pertussis, pDCs exhibit compromised maturation because of PTX. Simultaneously, the frequency of these pDCs producing IFN $\alpha$ was $\sim 3.5$-fold increased at this time but returns to baseline by $10 \mathrm{dpi}$ (Figure 4c). Though there is a modest increase in IFN $\alpha$-secreting pDCs in Bp $\triangle P T X$-infected mice, this change is not significantly different from the frequency in uninfected mice, suggesting that there are other toxins prompting IFN $\alpha$ secretion. However, lung IFN $\alpha$ mRNA at this time point is still significantly high (Figure 4d), indicating 
a

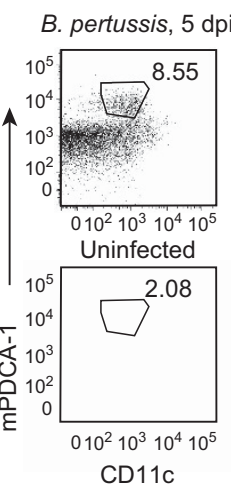

b

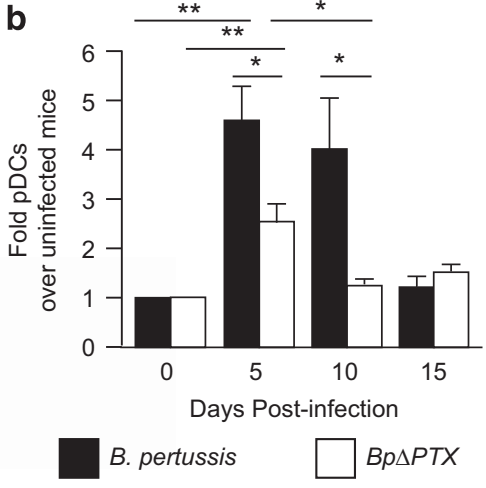

C Uninfected
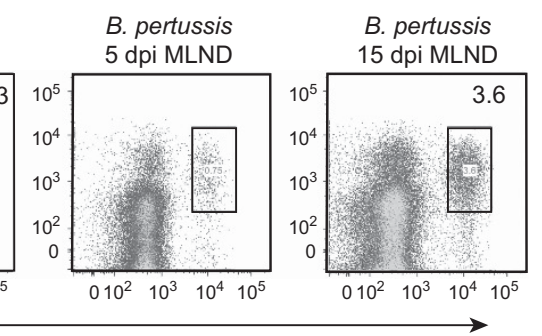

d

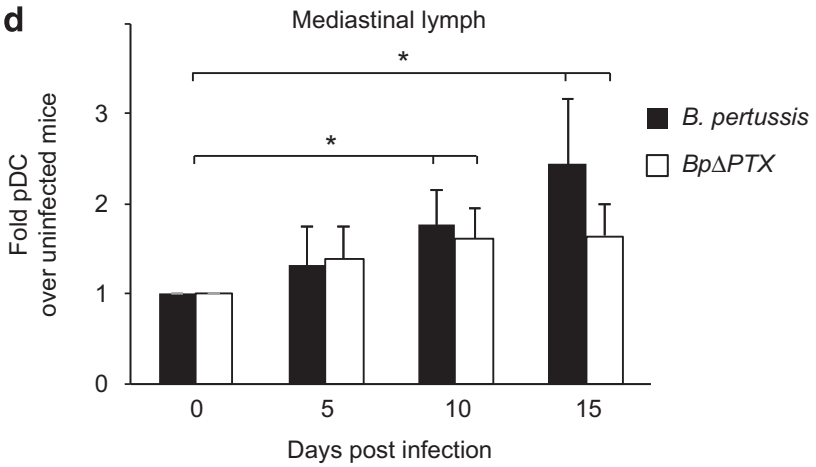

e
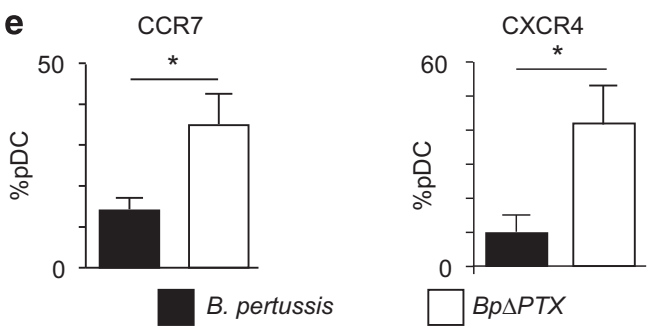

Figure 3 Temporal frequency and trafficking of pDCs. (a) pDCs were gated among enriched lung $\mathrm{CD} 11 \mathrm{c}^{+}$cells and characterized as CD11 $\mathrm{c}^{\text {int }}$, $\mathrm{CD}_{11 \mathrm{~b}^{-}}$, and $\mathrm{mPDCA}-1^{+}$. The frequency of pDCs from uninfected and $B$. pertussis-infected mice at 5 dpi was determined. (b) Frequency of pDCs in lungs from B. pertussis and Bp $\triangle P T X$-infected mice, expressed as fold over respective cells from uninfected mice. (c) The frequency of pDCs in mediastinal lymph nodes at various times post infection is depicted in representative flow cytometry plots. (d) Bar graph representing the percentage of pDCs in mediastinal lymph nodes of mice infected with $B$. pertussis or Bp $\triangle P T X$ at various dpi. (e) GPCR expression was analyzed on lung $\mathrm{pDC}$ s as detailed. The frequency of pDCs expressing CCR7 and CXCR4 in the lungs of mice at $10 \mathrm{dpi}$. (Bar graphs represent averages of at least three independent experiments; ${ }^{\star}{ }^{\star} P<0.01,{ }^{\star} P<0.05$.) dpi, days post-infection; GPCR, G protein-coupled receptor; pDCs, plasmacytoid dendritic cells.

that pDCs initially enrich the lungs with IFN $\alpha$, but other cells in the lungs most likely contribute to this environment as well in response to the infection.
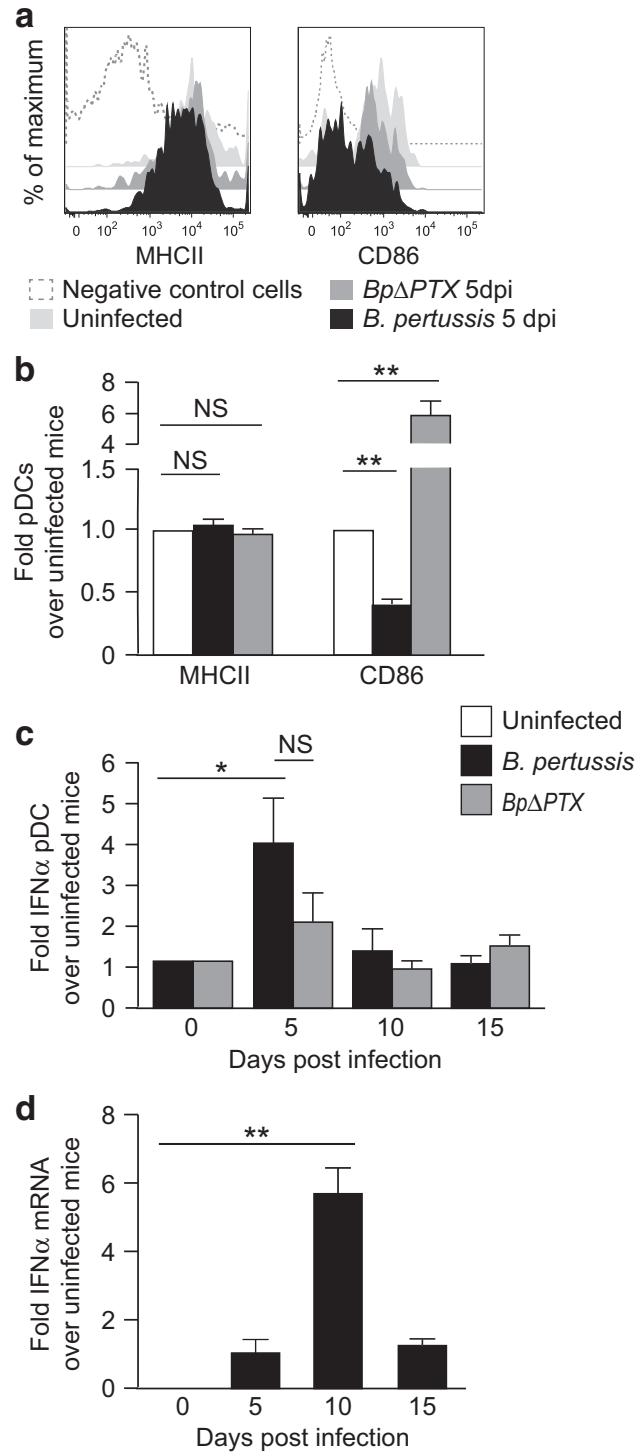

Figure 4 Phenotypic and functional characterization of lung pDCs during B. pertussis infection. CD11C ${ }^{+}$cells were collected from the lungs of

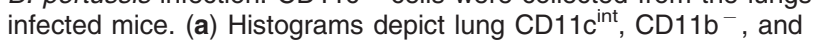
mPDCA $-1^{+}$cells (pDCs) at 5 dpi derived from B. pertussis, Bp $\triangle P T X$, and from uninfected mice. These were compared with $C D 11 c^{-}$and mPDCA$1^{-}$cells, which served as a negative control as described in the legend. (b) Summary bar graph representing the frequency of lung pDCs expressing maturation markers as depicted. Results are expressed as fold over pDC derived from uninfected mice. (c) pDCs were stained intracellularly for their secretion of IFN $\alpha$. Bar graph representation of the frequency of pDCs expressing IFN $\alpha$. Results are expressed as fold over uninfected mice. (d) mRNA expression of IFN $\alpha$ was measured in lungs at various times post infection as depicted. (Histograms represent one experiment from three independent experiments. Bar graphs represent averages from at least three independent experiments. ${ }^{\star} P<0.05$.) dpi, days post-infection; IFN, interferon; pDCs, plasmacytoid dendritic cells.

\section{IFN $\alpha$ reduces Th17 differentiation in vitro}

To test whether an IFN $\alpha$-rich environment limits Th17 differentiation by DCs, we used an allogeneic co-culture system where $\mathrm{CD} 11 \mathrm{c}^{+}$DCs from the lungs of 5 days- $B$. pertussis infected mice (Thy1.2) were cultured for 4 days with Thy1.1 $\mathrm{CD}^{+}{ }^{+}$cells at a ratio of 1:2 in Th17-polarizing conditions, with 

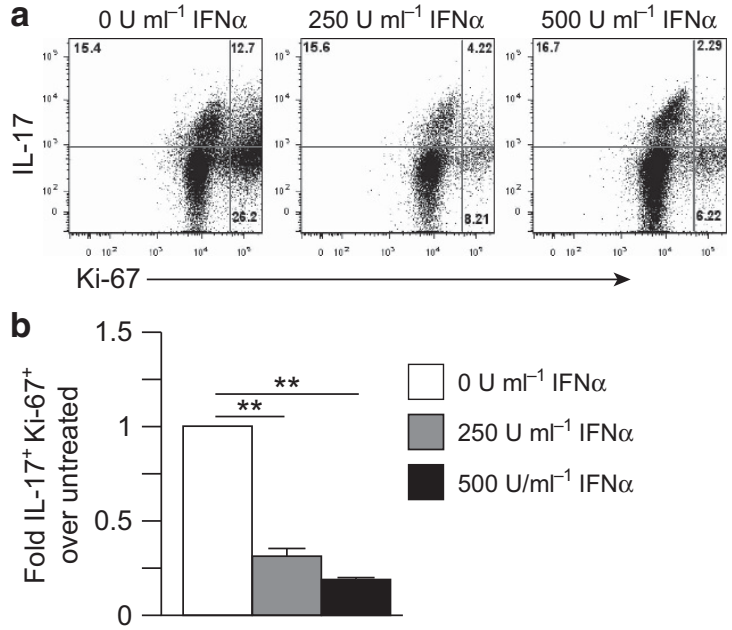

Figure 5 Effect of IFN $\alpha$ on Th17 differentiation by DC in vitro. CD $11 \mathrm{C}^{+}$cells from 5 days- $B$. pertussis infected mice (Thy $1.2^{+}$) were co-cultured for 4 days with purified $\mathrm{CD} 4^{+} \mathrm{T}$ cells from uninfected mice (Thy $1.1^{+}$) with Th17-polarizing conditions as described. IFN $\alpha$ was added at first day of co-culture at concentrations indicated. (a) Representative flow cytometry plot and gating strategy. (b) Bar graph of the frequency of proliferating Th17 cells (IL-17 $\left.{ }^{+}, \mathrm{Ki}-67^{+}\right)$. (Bar graph represents average of three independent experiments. ${ }^{* \star} P<0.01$, ${ }^{\star} P<0.05$.) IFN, interferon; IL, interleukin; NS, not statistically significant; Th, T helper.

varying concentrations of IFN $\alpha$. We then analyzed the frequency of $\mathrm{Ki}-67^{+}$proliferating, IL-17-producing cells with the addition of IFN $\alpha$ (Figure 5a). By plotting fold changes, we noted that the frequency of those cells dropped to 0.2 -fold compared with respective cells in non-polarizing conditions, which is about an $80 \%$ decrease due to the addition of IFN $\alpha$ (Figure 5b). These data reveal an inhibitory role for IFN $\alpha$, in vitro, on Th17 cell differentiation by lung DCs derived from B. pertussis-infected mice.

\section{In vivo blocking of $\mathrm{pDC}$-derived IFN $\alpha$ permits early rise of Th17 cells}

To ascertain whether the inhibitory effect of IFN $\alpha$ on Th17 differentiation in vitro is also evident in vivo, we treated mice with blocking antibodies that reflect diverse IFN $\alpha$ mechanisms: anti-SiglecH-blocks IFN $\alpha$ exclusively derived from $\mathrm{pDCs},{ }^{28}$ anti-PDCA-depletes mPDCA-1-expressing cells such as pDCs, (Figure 6a), ${ }^{29}$ and anti-type I IFN receptor (anti-INFAR)-blocks the general receptor for type I IFN on a variety of immune cells (Figure 6a).$^{30}$ We then assessed the frequency of Th17 cells and Th1 cells in the lungs at 2 and $5 \mathrm{dpi}$, when a prominent rise of Th17 was not previously observed.

At $2 \mathrm{dpi}$, no differences were observed in the frequency of Th1 cells between mice treated with all three blocking antibodies compared with their respective, isotype-controlantibody-injected mice (Figure $\mathbf{6 b}$ ). Similarly, Th17 cells in anti-PDCA- and anti-IFNAR-treated mice resembled the percentages seen in the respective isotype controls (Figure 6b and Supplementary Figure S1a online). However, the frequency of lung Th17 cells significantly increased in mice treated with anti-SiglecH at both 2 and 5 dpi (Figure 6c).
This confirms that IFN $\alpha$ inhibition of Th17 cells during $B$. pertussis infection is an early phenomenon related specifically to IFN $\alpha$ derived from $\mathrm{pDCs}$ and that complete depletion of pDCs (and perhaps additional PDCA-expressing cells during infection) or blocking broad type I IFN receptor (INFAR) across a variety of cells may interfere with Th17 differentiation. Furthermore, lung histology from mice treated with anti-SiglecH demonstrates a significant increase in cell recruitment at 2 dpi compared with the respective isotype control (Figure 6d and Supplementary Figure S1b). At the same time, only a modest increase of lung inflammation was observed in mice treated with anti-PDCA (Figure 6d), and minimal cell recruitment was observed in lung sections prepared from mice treated with anti-IFNAR (Supplementary Figure S1b). Taken together, these data imply that only specific blocking of IFN $\alpha$ derived from pDCs results in increased inflammation, perhaps owing to IL-17-mediated recruitment of neutrophils, Th17 cells, and additional immune cells.

Next, we investigated whether the increased frequency of Th17 cells and inflammation observed in the lungs during treatment with anti-SiglecH are reflected on the lung bacterial load. As the rise in Th17 cells was observed as early as $2 \mathrm{dpi}$, we assessed bacterial loads in the lungs at this time-point in all treated mice. Compared with isotype controls, only a moderate, but statistically insignificant, reduction in bacterial colony-forming units was observed in anti-PDCA-treated mice (Figure 7a), and no difference in bacterial load was observed in anti-IFNAR-treated mice (Supplementary Figure S1c). However, in the anti-SiglecH-treated mice, a significant reduction in the lung bacterial load was observed at $2 \mathrm{dpi}$ and even more at 5 dpi (Figure $7 \mathbf{a}$ and $\mathbf{b}$ ). Despite that, the bacterial loads in mice treated with anti-SiglecH and infected with B. pertussis for $10 \mathrm{dpi}$ converged with the isotype level (Figure 7b). A similar trend was observed when the experiment was extended to $15 \mathrm{dpi}$ (data not shown). This finding provides additional evidence that blocking IFN $\alpha$ secretion specifically from pDCs not only permits a rapid increase in Th17 cells, but also lessens the bacterial load during but only early post-infection.

\section{DISCUSSION}

Secretion of type I IFN is one of the earliest innate cytokine responses to pathogen invasion, leading to the activation and recruitment of immune cells. However, recent studies have shown that this response might impair early adaptive immune responses to some infections. ${ }^{20}$ In this study, we investigate whether or not pDCs and pDC-derived IFN $\alpha$ limits Th17 responses during $B$. pertussis infection, which may delay a timely anti-bacterial defense and impair resolution of whooping cough.

It is well-documented that Th17 cells have a role in the clearance of mucosal infections and, in particular, mediating protective immunity against $B$. pertussis. ${ }^{2,3,11,31,32}$ Here, we report that the emergence of these cells in mice infected with $B$. pertussis is primarily due to PTX, and demonstrate that Th17 cells appear late post-infection, and are sustained at least 

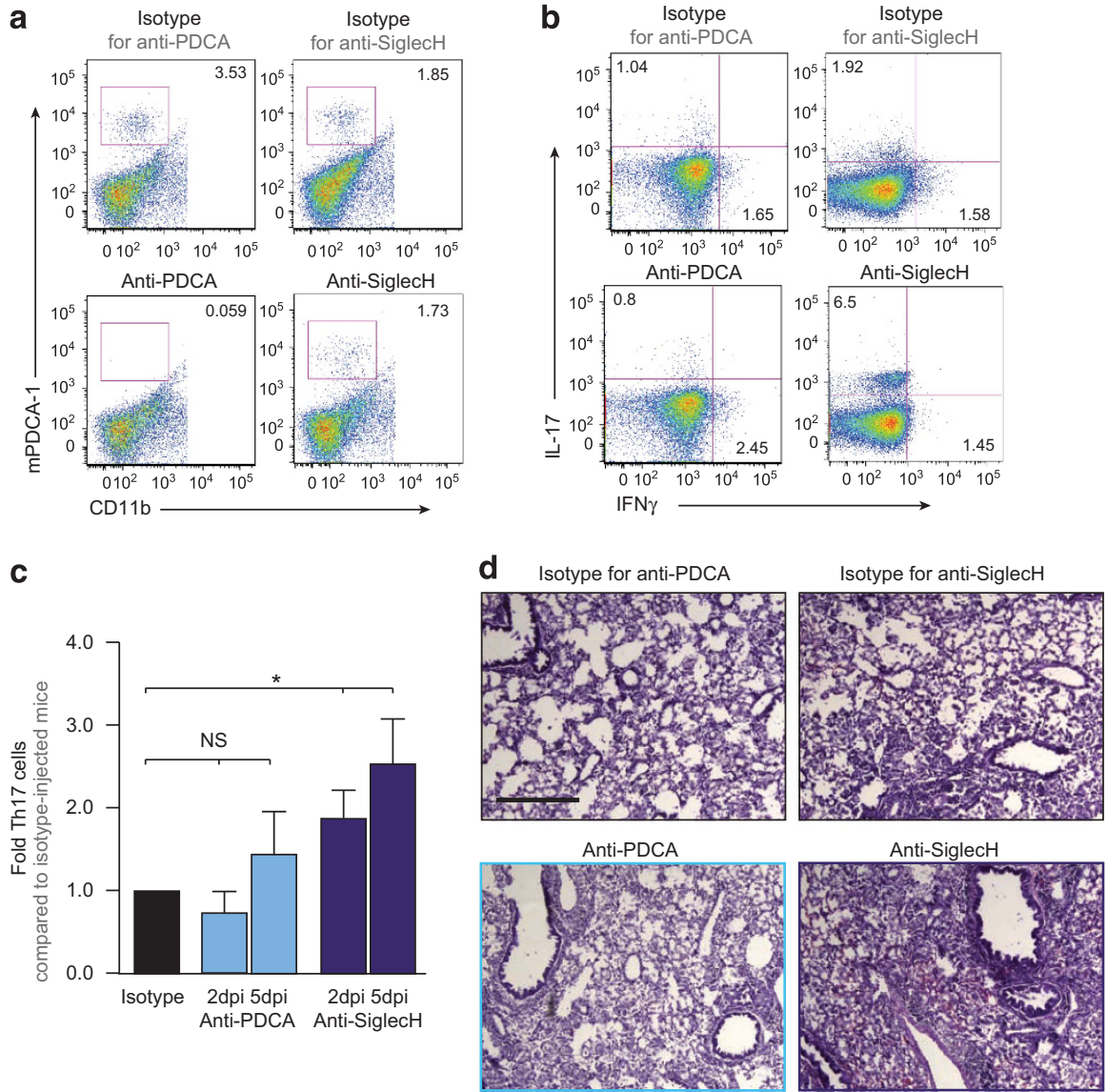

Figure 6 Anti-SiglecH but not anti-PDCA treatment increase early lung Th17 frequency and inflammation. Mice were treated with anti-SiglecH and anti-PDCA as explained in the MATERIALS AND METHODS section. (a) The frequency of pDCs was evaluated in the lungs following treatment with anti-PDCA at 2 dpi. (b) The frequency of lung CD4 ${ }^{+} \mathrm{CD}_{4} 4^{+}$cells expressing IL-17 and/or IFN $\gamma$ derived from mice treated with anti-SiglecH, antiPDCA, or respective isotypes was determined at 2 dpi by flow cytometry. (c) The frequency of lung CD4 ${ }^{+}$CD $44^{+}$cells expressing IL- 17 from mice treated with anti-SiglecH, anti-PDCA, or respective isotype at 2 and $5 \mathrm{dpi}$ and expressed as the fold over respective isotype-treated mice. (d) $\mathrm{H} \& \mathrm{E}$ staining of lung sections from mice treated with anti-SiglecH, anti-PDCA, or respective isotype at $2 \mathrm{dpi}$. At least 100 fields were analyzed; scale bar $=400 \mu \mathrm{m}$. (Bar graph represents average of three independent experiments. ${ }^{*} P<0.05$.) dpi, days post-infection; H\&E, hematoxylin and eosin; IFN, interferon; IL, interleukin; NS, not statistically significant; pDCs, plasmacytoid dendritic cells; Th, T helper.

until 50 dpi. Although Th17 cells are recognized as adaptive immune response cells, recent studies demonstrate that infected mice can mount Th17 and IL-17 responses as early as 2-3 dpi. Examples include mice infected with Candida albicans, and Klebsiella pneumonia. ${ }^{33-35}$ This implies that the emergence of Th17 cells in B. pertussis-infected mice is somehow inhibited. As the late appearance of Th17 cells was paralleled in both the blood and lungs of infected mice, we speculate that the delay might be associated with the inhibition of differentiation rather than impaired lung recruitment of Th17 cells.

Recent reports on the inhibitory effects of type I IFN on IL-17 responses, ${ }^{18-21}$ and evidence indicating a type I IFN response triggered by $B$. pertussis-filamentous hemagglutinin in peripheral blood mononuclear cells, ${ }^{22}$ led us to ask whether the delay in Th17 cells may be attributed to IFN $\alpha$. We focused our study on pDCs, which are prominent secretors of type I IFNs in response to pathogenic molecules binding to cytosolic TLRs. Although B. pertussis is considered an extracellular pathogen, several studies have documented internalization within myeloid cells and survival in lysosomal compartments as a strategy for the bacterium to evade host defense. ${ }^{36-38}$ Thus, in these circumstances, bacterial DNA may activate TLR9 in myeloid cells. Moreover, B. pertussis has been shown to use a type III secretion system to inject effector proteins into the eukaryotic cytosol. Respiratory infection with a type III secretion system mutant induced significantly higher IL-17 responses, indicating that the type III secretion system mechanism can introduce proteins that inhibit them. ${ }^{39}$

We show a sharp increase in the number of IFN $\alpha$-expressing pDCs in the lungs of mice, early post- $B$. pertussis infection. We attribute this increase not only to the enhanced recruitment of pDCs to the infected lungs, but also to PTX-dependent reduced migration of pDCs into the lymphatics, due to decreased frequency of $\mathrm{pDCs}$ expressing $\mathrm{G}$ protein-coupled receptor. The accumulation of pDCs in the lung of B. pertussis-infected mice, therefore, creates a rich environment in IFN $\alpha$ that may alter lung DC capacity to drive T-cell differentiation. ${ }^{40}$

Other studies have demonstrated that IFN $\alpha$-treated antigenpresenting cells exacerbated the differentiation of Th17 cells 

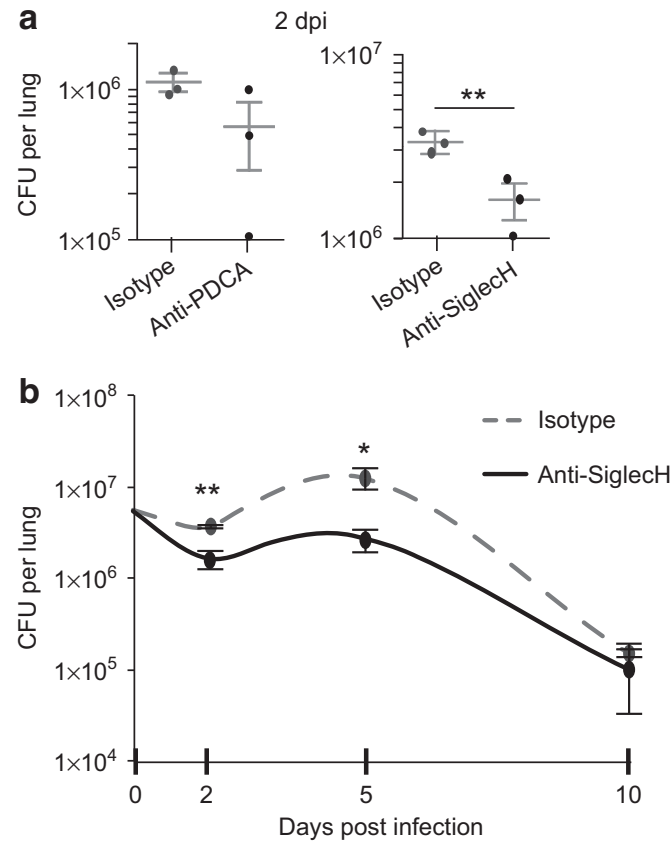

Figure 7 Lung bacterial loads in $B$. pertussis-infected mice treated with blocking antibodies. (a) The CFU of bacteria per lung was calculated and averaged at $2 \mathrm{dpi}$. (b) Timeline of lung bacterial loads in mice treated with anti-SiglecH or isotype control and infected with B. pertussis. The CFU of bacteria per lung was calculated and averaged at respective time points as depicted. At least three mice were used in each experiment. (Bar graph represents average of three mice for 2 and $5 \mathrm{dpi}$ and two mice for $10 \mathrm{dpi}$. ${ }^{*} P<0.05$.) CFU, colony-forming unit; dpi, days post-infection; NS, not statistically significant.

triggered by IL-6 and TGF $\beta$ through an IL-27/STAT1 mechanism. $^{41-44}$ Moreover, it has been reported that IFN $\alpha$ suppresses the synthesis of IL-17 in peripheral blood mononuclear cells by limiting the expression of TGF $\beta$ in macrophages. ${ }^{45,46}$ In an experimental autoimmune encephalomyelitis mouse model, it was shown that type I IFN promotes IL-10 secretion by $\mathrm{CD} 4{ }^{+}$cells while suppressing IL-17. ${ }^{21}$ Similarly, we show in an allogeneic system, which includes lung DCs derived from $B$. pertussis-infected mice, that the addition of IFN $\alpha$ resulted in a reduced capacity of Th17 differentiation. These results strongly imply that IFN $\alpha$ can compromise Th17-cell differentiation and perhaps promote suppressor $\mathrm{T}$ regulatory cells.

In line with these results, blocking $\mathrm{pDC}$-derived $\mathrm{IFN} \alpha$, in vivo, led to an increased frequency of Th17 cells in lungs of mice infected with $B$. pertussis as early as 2 and $5 \mathrm{dpi}$. These mice also exhibited inflammation and a reduced bacterial load in the lungs at this time. The results in this study do not rule out the possibility that the rapid bacterial resolution and lung inflammation observed in mice treated with IFN $\alpha$-blocking antibody may also reflect a removed suppression of IFN $\alpha$ on innate IL-17 immune responses, such as that from $\gamma \delta$ T cells.

Surprisingly, the results obtained in vivo with anti-SiglecH treatment were not replicated in mice depleted of pDCs (with anti-PDCA) nor in mice with the broad range type I IFN receptor blocked (with anti-IFNAR). Although mPDCA-1 is primarily expressed on pDCs in homeostatic conditions, in response to infection and IFN $\alpha$, other cells, such as B cells and conventional DCs, upregulate this marker. ${ }^{16,47}$ In addition, pDCs have been shown to contribute to the differentiation of T cells. ${ }^{48}$ Therefore treatment with anti-PDCA post infection may deplete cells, besides pDCs, that affect T-cell differentiation. $^{29}$ Also, numerous studies document the depletion of pDCs, and other cells as well, in mice that lack type I IFN receptor in a disease condition. ${ }^{49}$ This further supports the emerging data that interference with type I IFN receptors hinders cells' ability to drive T-cell differentiation.

The rise in lung Th17 cells following treatment with anti-SiglecH occurs rapidly, post infection, and is very specific to IFN $\alpha$ blocking. Coinciding with this observation, the reduction in the lung bacterial load following treatment with anti-SiglecH pertains to early stages of infection. The additional daily treatment with this blocking antibody up to 10 and $15 \mathrm{dpi}$ does not further increase the frequency of Th17 cells in the lungs over the robust response from natural infection at this time (data not shown). At these times post infection, we also do not see a further reduced bacterial load compared with non-treated mice. Perhaps with an early administration of anti-SiglecH in combination with additional antibacterial drugs, it would be possible to induce a faster and more robust Th17 response that will attenuate or prevent the bacterial colonization. This possibility should be carefully verified in the future as a potential approach to treat whooping cough and induce a specific and fast Th17 immune response to a vaccine.

The role of pDCs in B. pertussis pathogenesis, as we understand it, is depicted in Figure 8. We suggest that infection of mice with $B$. pertussis leads to rapid recruitment of immature, IFN $\alpha$-secreting $\mathrm{pDCs}$ in the lungs. These $\mathrm{pDCs}$ rapidly accumulate because of a PTX-dependent inhibition of tissue exit to the lymphatics, and contribute to an IFN $\alpha$-rich environment. Such an environment impedes Th17 differentiation by DCs, leading to a delayed IL-17 response and bacterial resolution. Therefore, blocking pDC-derived IFN $\alpha$ permits early Th17 differentiation, an increased recruitment and inflammation, and a reduced bacterial load early in the lungs. The experimental design presented here demonstrates an early effect, which is also specific for the IFN $\alpha$ derived from $\mathrm{pDCs}$. This also suggests a critical role for $\mathrm{pDCs}$ in Th17 differentiation, because the complete elimination of these cells does not allow the early rise in Th17.

To the best of our knowledge, this study is the first to suggest a role for $\mathrm{pDCs}$, specifically $\mathrm{pDC}$-derived IFN $\alpha$, in $B$. pertussis pathogenesis. The results in this study not only provide a more precise understanding of the roles of $\mathrm{pDCs}$ and IFN $\alpha$ during $B$. pertussis infection, but also expand our understanding of immunological responses during respiratory infections.

\section{MATERIALS AND METHODS}

Animals. Animal experiments were handled following the guidelines approved by the Institutional Animal Care and Use Committee 


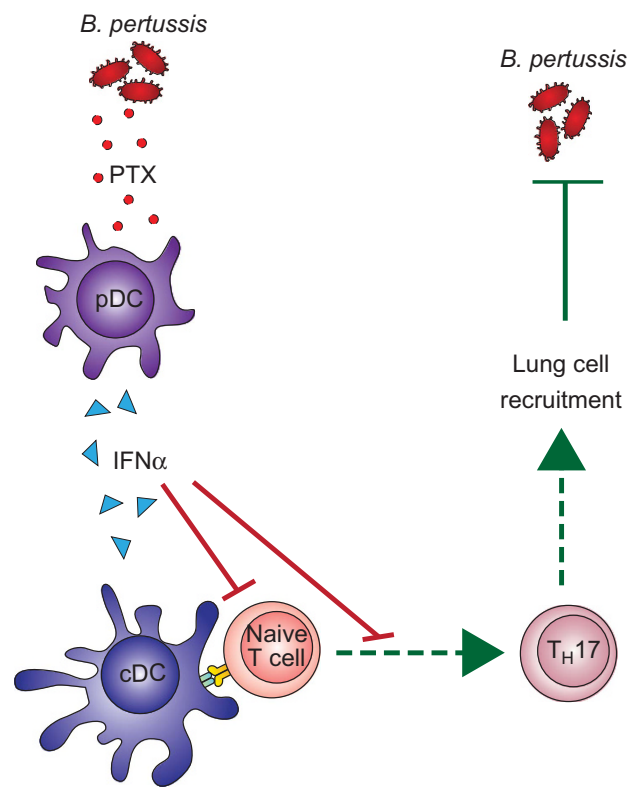

Figure 8 Model of pDC modulation on Th17 differentiation during $B$. pertussis infection. $\mathrm{pDC}$ accumulation and secretion of IFN $\alpha$ in the lungs inhibit Th17 differentiation during infection, limiting an early appearance of Th17 cells, and compromises resolution of $B$. pertussis early during infection. IFN, interferon; pDC, plasmacytoid dendritic cell; Th, T helper.

(IACUC; protocol \#921). Female BALB/c (Thy1.2 ${ }^{+}$) mice and female FVB/N $\left(\right.$ Thy $1.1^{+}$) mice were purchased from Simonsen Labs (Gilroy, CA).

Bacterial strains and growth conditions. $B$. pertussis strains used in this study were nalidixic acid-resistant B. pertussis 338 and Bp $\triangle P T X$ (ptxD6), which is a derivative of parental strain $B p 338$ with a deletion of the PTX operon and a substitution of a kanamycin-resistance cassette at that location. ${ }^{50}$ B. pertussis strains were grown on BordetGengou blood agar plates.

Infection. Inocula were prepared by streaking bacterial strains from frozen culture on Bordet-Gengou blood agar plates. After growth for 3 days at $37^{\circ} \mathrm{C}$, bacteria were transferred to new plates and grown for an additional 3 days. Bacterial strains were resuspended in sterile phosphate-buffered saline (PBS). Six-week-old female BALB/c mice were anesthetized by inhalation of isoflurane and administered intranasal with 25 ul of $5 \times 10^{6}$ colony-forming unitsof either $B$. pertussis or $B p \triangle P T X$ or PBS. Mice were killed by inhalation of isoflurane at various times post infection, and tissues were collected for experimentation. Lungs from experimentally infected mice were homogenized and plated on Bordet-Gengou plates to assess bacterial load.

Digestion of lung tissue. Lungs from infected or control BALB/c mice were perfused with $30-50 \mathrm{ml}$ of PBS. The lungs and trachea were removed, processed, and digested in RPMI-1640 media containing $10 \%$ fetal calf serum, $75 \mathrm{U} \mathrm{ml}^{-1}$ DNase I (Sigma-Aldrich, St Louis, $\mathrm{MO}$ ) and $250 \mathrm{U} \mathrm{ml}^{-1}$ Type I collagenase (Worthington Biochemical, Lakewood, NJ) in a $37^{\circ} \mathrm{C}$ orbital shaker for $45-60 \mathrm{~min}$ (5 ml media per lung). The digested lungs were passed through a $70-\mu \mathrm{m}$ nylon mesh strainer, and cells were washed with RPMI-1640 to remove residual digest medium.

Isolation of lung mononuclear cells. Digested lung tissue was resuspended in $40 \%$ Percoll (GE Lifescience, Piscataway, NJ), slowly underlaid with $70 \%$ Percoll, and centrifuged at $300 \times g$ for $25 \mathrm{~min}$ (without brakes) to create a density gradient. Cells at the $40-70 \%$ interface (mononuclear cells) were collected and stained for flow cytometry analysis.

Preparation of blood leukocytes. Whole blood from infected or control mice was obtained by cardiac puncture and mixed with a solution containing $1.5 \mathrm{~mm}$ EDTA and 2\% dextran, and incubated at $37^{\circ} \mathrm{C}$ for $30 \mathrm{~min}$. Leukocyte-rich plasma, located on the top of the aggregated erythrocytes, was carefully collected and centrifuged at $300 \times g$ for $5 \mathrm{~min}$. The cell pellets were mixed with $3 \mathrm{ml}$ red blood cell lysis buffer for $5 \mathrm{~min}$ and washed with RPMI-1640 containing 10\% fetal calf serum.

Cell enrichment. All conjugated magnetic microbeads, LD or LS column, and QuadroMacs separator were used according to the manufacturer's instructions and purchased from Miltenyi Biotech, Auburn, CA.

Enrichment of $\mathrm{CD}_{11 c^{+}}$cells in the lungs. Digested lung tissue was enriched for $\mathrm{CD}_{11 \mathrm{c}^{+}}$cells by positive selection using anti-CD11c ${ }^{+}$ magnetic microbeads and LD column.

Enrichment of $\mathrm{CD}_{11 \mathrm{c}^{+}}$cells in the mediastinal lymph nodes. The mediastinal lymph nodes were harvested, pooled for each mouse, and pressed through a nylon mesh to create a single cell suspension. Cells were labeled with anti-CD11c microbeads and positively selected using a LD column.

Enrichment of CD4 ${ }^{+}$cells spleen. Splenocytes obtained from female $\mathrm{FVB} / \mathrm{N}$ Thy1.1 + mice, were enriched for $\mathrm{CD} 4^{+} \mathrm{T}$ cells using antiCD4 microbeads, positively selected using a LS column.

T-cell activation and co-culture experiments. Lung $\mathrm{CD} 11 \mathrm{c}^{+}$and spleen CD4 + cells from FVB/N Thy1.1 + mice were cultured at 1:2 ratio in a 96-well flat-bottom plate in 200 ul of RPMI 1640 supplemented with $10 \%$ fetal calf serum, $1 \%$ penicillin, and $50 \mu \mathrm{M} 2$ mercaptoethanol in the presence or absence of $5 \mu \mathrm{g} \mathrm{ml}{ }^{-1}$ anti-CD3 Ab (clone 2C11; Biolegend, San Diego, CA) and $1 \mu \mathrm{g} \mathrm{ml}^{-1}$ anti-CD28 (clone 37.51; Biolegend). The exogenous cytokines used for Th17 polarization were $50 \mathrm{ng} \mathrm{ml}^{-1} \mathrm{IL}-6$ (R\&D Systems, Minneapolis, MN), $1 \mathrm{ng} \mathrm{ml}^{-1}$ TGF $\beta$ (R\&D Systems), and IFN $\alpha$ (PBL Assay Science, Piscataway, NJ). Cells were cultured for 4 days at $37^{\circ} \mathrm{C}$ with $5 \% \mathrm{CO}_{2}$ to allow for T-cell proliferation and Th17 differentiation and subsequently analyzed via flow cytometry.

Flow cytometry. For the T-cell experiments, lung mononuclear cells were stimulated with $20 \mathrm{ng} \mathrm{ml}^{-1}$ of Phorbol 12-myristate 13-acetate (PMA) (Sigma-Aldrich, St Louis, MO), $1 \mu \mathrm{g} \mathrm{ml}^{-1}$ of ionomycin, and $1 \mu \mathrm{lml}{ }^{-1}$ of Brefeldin A (BioLegend) for $4-6 \mathrm{~h}$ at $37^{\circ} \mathrm{C}$ with $5 \% \mathrm{CO}_{2}$. Cells were stained with extracellular staining antibodies, and then stained intracellular with the BD Transcription Factor Buffer Set (BD Biosciences, San José, CA) according to directions from the manufacturer. Antibodies used for the T-cell stain were as follows: IL-17 (TC11-18H10.1, CD4 (RM4-5), CD44 (IM7), and IFN $\gamma$ (XMG1.2), CD29( $\beta 1)$ (HMB1-1), CD49d ( $\alpha 4)(9 \mathrm{C} 10)$, beta7 FIB27, CD90.1 (OX7), and Ki-67 (16A8). For the pDC experiments, pDCs were stimulated with $12 \mathrm{~mm}$ of the TLR7 agonist, Imiquimod (Santa Cruz Biotech, Santa Cruz, CA), for $36 \mathrm{~h}$, with $1 \mu \mathrm{l} \mathrm{ml}{ }^{-1}$ of Brefeldin A (BioLegend) in the last $12 \mathrm{~h}$, not exceeding $12 \mathrm{~h}$. Cells were then stained with extracellular staining antibodies, and then fixed by incubating in $200 \mu \mathrm{l}$ of $4 \%$ paraformaldehyde (in PBS) for $20 \mathrm{~min}$. Cells were permeabilized with $0.1 \%$ saponin (in PBS), and then stained intracellular with intracellular antibodies. Antibodies used for the pDC stain were as follows: CD11c (N418), mPDCA-1 (927), CD11b (M1-70), CCR7 (4B12), MHC II (M5/114.15.2), CD86 (GL1, BD Biosciences), CXCR4 (2B11 eBioscience, San Diego, CA), IFN $\alpha$ (RMMA-1,PBL Assay Science). All antibodies were purchased from BioLegend, unless otherwise stated. Flow cytometry analysis was performed on either the FACSCantoII (BD Biosciences) or FACSLSRII (BD Biosciences), and data were analyzed with FlowJo software (Tree Star, Ashland, OR). 
In vivo $\mathrm{pDC}$ depletions. $\mathrm{BALB} / \mathrm{c}$ mice were injected, intraperitoneally, with functional-grade purified anti-PDCA (927) (BioLegend or a kind gift contributed by Dr. Eugene Butcher from Stanford University and Dr. Marco Colonna from Washington University in St Louis), anti-SiglecH (440c) (Bio X Cell, West Lebanon, NH), IgG2b isotype (LTF-2) (Bio X Cell), anti-IFNAR (MAR1-5A3) (BioLegend), or mouse anti-human IFNGR1 (GIR-208) isotype (BioLegend). We injected $100 \mu \mathrm{g}$ in the anti-SiglecH, IgG2b Isotype, and anti-PDCA studies. Anti-SiglecH and IgG2b Isotype injections began 2 days prior to and throughout infection, and anti-PDCA injections were only administered for 2 days prior to infection. For the anti-IFNAR studies, we began 2-mg injections 1 day prior to infection and continued with $1-\mathrm{mg}$ injections throughout infection.

Histology. Mice were killed at dpi 2 and 4 . Lungs were perfused with $1 \times \mathrm{PBS}$ and fixed in $4 \%$ paraformaldehyde (in $1 \times \mathrm{PBS}$ ) at $4{ }^{\circ} \mathrm{C}$ overnight, then transferred to $30 \%$ sucrose (in $1 \times \mathrm{PBS}$ ) at $4{ }^{\circ} \mathrm{C}$ until they sank ( $\sim$ 4days). Tissue was embedded in Tissue-Tek O.C.T. Compound (Fisher Scientific, Pittsburg, PA), flash-frozen, and stored at $-80^{\circ} \mathrm{C}$ until sliced into $8-\mu \mathrm{m}$ sections using a cryostat Leica (Buffalo Grove, IL). A classic hematoxylin and eosin stain was performed and images were acquired with a Leica microscope (DMI 4000 B) and camera (DFC310 FX).

RT-PCR analysis. Total RNA was isolated using the RNeasy mini kit (Qiagen, Courtaboeuf, France). RNA (1 ug) was reverse-transcribed using the RETROscript (Ambion, Foster City, CA). The analysis of mRNA expression level was assessed with SYBR Green I mastermix using the ABI 7100 software and the following primers: IL-17A forward, 5'-ACCTCAACCGTTCCACGTCA-3'; IL-17A reverse, $5^{\prime}$-CAGGGTCTTCATTGCGGTG-3'; IFN $\alpha$ forward, $5^{\prime}$-GCAACCC TCCTAGACTCATTCT- $3^{\prime}$; IFN $\alpha$ reverse, $5^{\prime}$-CCAGCAGGGCGTCT TCCT-3'; GAPDH forward, 5' AGGTCGGTGTGAACGGATTTG 3'; GAPDH reverse, $5^{\prime}$ TGTAGACCATGTAGTTGAGGTCA $3^{\prime}$. Reactions were run in triplicates and normalized to endogenous GAPDH mRNA level using the $2-\delta C t$ method. Specific mRNA levels were expressed as fold increase over the uninfected condition.

Statistical analysis. Data are presented as mean values \pm standard error of the mean (s.e.m.). Statistical significance between sets of data was assessed with the two-tailed Student's $t$-test.

SUPPLEMENTARY MATERIAL is linked to the online version of the paper at http://www.nature.com/mi

\section{ACKNOWLEDGMENTS}

We thank Katharina Lahl and Eugene Butcher (Stanford University) for the donation of blocking antibodies and for the use of the LSRII and their critical advice. We also acknowledge the following students who assisted at various stages of this work: Joseph Anderson, Jennifer Stokes, Elvia Silva, Sreelakshmi V Maroli, Tarek Jakoush, Edgar Henriquez and Christopher Sequeira. This work was supported by NIH-SC3GM100854 to TA and also by $\mathrm{NIH}$-Maximizing Access to Research Careers (MARC) funds to RF and $\mathrm{NIH}-$ Research Initiative for Scientific Enhancement (RISE) funds to PL.

\section{DISCLOSURE}

The authors declare no conflict of interest.

c) 2016 Society for Mucosal Immunology

\section{REFERENCES}

1. Higgs, R., Higgins, S.C., Ross, P.J. \& Mills, K.H. Immunity to the respiratory pathogen Bordetella pertussis. Mucosal Immunol. 5, 485-500 (2012).

2. Warfel, J.M. \& Merkel, T.J. Bordetella pertussis infection induces a mucosal IL-17 response and long-lived Th17 and Th1 immune memory cells in nonhuman primates. Mucosal Immunol. 6, 787-796 (2013).
3. Warfel, J.M., Zimmerman, L.I. \& Merkel, T.J. Acellular pertussis vaccines protect against disease but fail to prevent infection and transmission in a nonhuman primate model. Proc. Natl Acad. Sci. USA 111, 787-792 (2014).

4. Fedele, G. et al. Bordetella pertussis commits human dendritic cells to promote a Th1/Th17 response through the activity of adenylate cyclase toxin and MAPK-pathways. PLoS One 5, e8734 (2010).

5. Adkins, l. et al. Bordetella adenylate cyclase toxin differentially modulates toll-like receptor-stimulated activation, migration and T cell stimulatory capacity of dendritic cells. PLoS One 9, e104064 (2014).

6. Dunne, A. et al. Inflammasome activation by adenylate cyclase toxin directs Th17 responses and protection against Bordetella pertussis. J. Immunol. 185, 1711-1719 (2010).

7. Fedele, G. et al. Lipopolysaccharides from Bordetella pertussis and Bordetella parapertussis differently modulate human dendritic cell functions resulting in divergent prevalence of Th17-polarized responses. J Immunol. 181, 208-216 (2008).

8. Stenger, R.M. et al. Immunodominance in mouse and human CD4 + T-cell responses specific for the Bordetella pertussis virulence factor P.69 pertactin. Infect. Immun. 77, 896-903 (2009).

9. Scanlon, K.M. et al. Epithelial anion transporter pendrin contributes to inflammatory lung pathology in mouse models of Bordetella pertussis infection. Infect. Immun. 82, 4212-4221 (2014).

10. Andreasen, C., Powell, D.A. \& Carbonetti, N.H. Pertussis toxin stimulates IL-17 production in response to Bordetella pertussis infection in mice. PLoS One 4, e7079 (2009).

11. Andreasen, C. \& Carbonetti, N.H. Role of neutrophils in response to Bordetella pertussis infection in mice. Infect. Immun. 77, 1182-1188 (2009).

12. Higgins, S.C., Jarnicki, A.G., Lavelle, E.C. \& Mills, K.H. TLR4 mediates vaccine-induced protective cellular immunity to Bordetella pertussis: role of IL-17-producing T cells. J. Immunol. 177, 7980-7989 (2006).

13. Henderson, M.W. et al. Contribution of Bordetella filamentous hemagglutinin and adenylate cyclase toxin to suppression and evasion of IL-17mediated inflammation. Infect. Immun. 80, 2061-2075 (2012).

14. Dubin, P.J. \& Kolls, J.K. Th17 cytokines and mucosal immunity. Immunol. Rev. 226, 160-171 (2008).

15. Colonna, M., Trinchieri, G. \& Liu, Y.J. Plasmacytoid dendritic cells in immunity. Nat. Immunol. 5, 1219-1226 (2004).

16. Asselin-Paturel, C. \& Trinchieri, G. Production of type I interferons: plasmacytoid dendritic cells and beyond. J. Exp. Med. 202, 461-465 (2005).

17. Parker, D. \& Prince, A. Type I interferon response to extracellular bacteria in the airway epithelium. Trends Immunol. 32, 582-588 (2011).

18. Henry, T. et al. Type I IFN signaling constrains IL-17A/F secretion by gammadelta $\mathrm{T}$ cells during bacterial infections. J. Immunol. 184, 3755-3767 (2010).

19. Nikolaus, S. et al. Interferon beta-1a in ulcerative colitis: a placebo controlled, randomised, dose escalating study. Gut 52, 1286-1290 (2003).

20. Tilg, $\mathrm{H}$. et al. A randomised placebo controlled trial of pegylated interferon alpha in active ulcerative colitis. Gut 52, 1728-1733 (2003).

21. Zhang, L., Yuan, S., Cheng, G. \& Guo, B. Type I IFN promotes IL-10 production from $T$ cells to suppress Th17 cells and Th17-associated autoimmune inflammation. PLoS One 6, e28432 (2011).

22. Dieterich, C. \& Relman, D.A. Modulation of the host interferon response and ISGylation pathway by B. pertussis filamentous hemagglutinin. PLoS One 6, e27535 (2011).

23. Nguyen, T.M. et al. Differential expression of alpha 4 integrins on effector memory $T$ helper cells during Bordetella infections. Delayed responses in Bordetella pertussis. PLoS One 7, e52903 (2012).

24. Penna, G., Sozzani, S. \& Adorini, L. Cutting edge: selective usage of chemokine receptors by plasmacytoid dendritic cells. J. Immunol. 167, 1862-1866 (2001).

25. Yoneyama, $\mathrm{H}$. et al. Evidence for recruitment of plasmacytoid dendritic cell precursors to inflamed lymph nodes through high endothelial venules. Int. Immunol. 16, 915-928 (2004).

26. Eppert, B.L., Motz, G.T., Wortham, B.W., Flury, J.L. \&Borchers, M.T. CCR7 deficiency leads to leukocyte activation and increased clearance in response to pulmonary Pseudomonas aeruginosa infection. Infect. Immun. 78, 2099-2107 (2010). 
27. Umemoto, E. et al. Constitutive plasmacytoid dendritic cell migration to the splenic white pulp is cooperatively regulated by CCR7- and CXCR4mediated signaling. J. Immunol. 189, 191-199 (2012).

28. Blasius, A.L. \& Colonna, M. Sampling and signaling in plasmacytoid dendritic cells: the potential roles of Siglec-H. Trends Immunol. 27, 255-260 (2006).

29. Blasius, A.L., Giurisato, E., Cella, M., Schreiber, R.D., Shaw, A.S. \& Colonna, M. Bone marrow stromal cell antigen 2 is a specific marker of type I IFN-producing cells in the naive mouse, but a promiscuous cell surface antigen following IFN stimulation. J. Immunol. 177, 3260-3265 (2006).

30. Sheehan, K.C. et al. Blocking monoclonal antibodies specific for mouse IFN-alpha/beta receptor subunit 1 (IFNAR-1) from mice immunized by in vivo hydrodynamic transfection. J. Interferon Cytokine Res. 26, 804-819 (2006).

31. Ross, P.J. et al. Relative contribution of Th1 and Th17 cells in adaptive immunity to Bordetella pertussis: towards the rational design of an improved acellular pertussis vaccine. PLoS Pathog. 9, e1003264 (2013).

32. Kolls, J.K. \& Khader, S.A. The role of Th17 cytokines in primary mucosal immunity. Cytokine Growth Factor Rev. 21, 443-448 (2010).

33. Ye, P. et al. Interleukin-17 and lung host defense against Klebsiella pneumoniae infection. Am. J. Respir. Cell Mol. Biol. 25, 335-340 (2001).

34. Hernandez-Santos, N., Huppler, A.R., Peterson, A.C., Khader, S.A., McKenna, K.C. \& Gaffen, S.L. Th17 cells confer long-term adaptive immunity to oral mucosal Candida albicans infections. Mucosal Immunol. 6, 900-910 (2013).

35. Huang, W., Na, L., Fidel, P.L. \& Schwarzenberger, P. Requirement of interleukin-17A for systemic anti-Candida albicans host defense in mice. J. Infect. Dis. 190, 624-631 (2004).

36. Ewanowich, C.A., Melton, A.R., Weiss, A.A., Sherburne, R.K. \& Peppler, M.S. Invasion of HeLa 229 cells by virulent Bordetella pertussis. Infect. Immun. 57, 2698-2704 (1989).

37. Friedman, R.L., Nordensson, K., Wilson, L., Akporiaye, E.T. \& Yocum, D.E. Uptake and intracellular survival of Bordetella pertussis in human macrophages. Infect. Immun. 60, 4578-4585 (1992).

38. Lamberti, Y., Gorgojo, J., Massillo, C. \& Rodriguez, M.E. Bordetella pertussis entry into respiratory epithelial cells and intracellular survival. Pathog. Dis. 69, 194-204 (2013).

39. Fennelly, N.K. et al. Bordetella pertussis expresses a functional type III secretion system that subverts protective innate and adaptive immune responses. Infect. Immun. 76, 1257-1266 (2008).
40. Manh, T.P., Alexandre, Y., Baranek, T., Crozat, K. \& Dalod, M. Plasmacytoid, conventional, and monocyte-derived dendritic cells undergo a profound and convergent genetic reprogramming during their maturation. Eur. J. Immunol. 43, 1706-1715 (2013).

41. Guo, B., Chang, E.Y. \& Cheng, G. The type I IFN induction pathway constrains Th17-mediated autoimmune inflammation in mice. J. Clin. Invest. 118, 1680-1690 (2008).

42. Diveu, C. et al. IL-27 blocks RORc expression to inhibit lineage commitment of Th17 cells. J. Immunol. 182, 5748-5756 (2009).

43. Amadi-Obi, A. et al. TH17 cells contribute to uveitis and scleritis and are expanded by IL-2 and inhibited by IL-27/STAT1. Nat. Med. 13, 711-718 (2007).

44. Stumhofer, J.S. et al. Interleukin 27 negatively regulates the development of interleukin 17-producing $T$ helper cells during chronic inflammation of the central nervous system. Nat. Immunol. 7, 937-945 (2006).

45. Moschen, A.R., Geiger, S., Krehan, I., Kaser, A. \& Tilg, H. Interferon-alpha controls IL-17 expression in vitro and in vivo. Immunobiology 213 , 779-787 (2008).

46. Dhanani, S., Huang, M., Wang, J. \& Dubinett, S.M. Interferon-alpha inhibits murine macrophage transforming growth factor-beta mRNA expression. Inflammation 18, 301-309 (1994).

47. Asselin-Paturel, C., Brizard, G., Pin, J.J., Briere, F. \& Trinchieri, G. Mouse strain differences in plasmacytoid dendritic cell frequency and function revealed by a novel monoclonal antibody. J. Immunol. 171, 6466-6477 (2003).

48. Boonstra, A. et al. Flexibility of mouse classical and plasmacytoid-derived dendritic cells in directing $T$ helper type 1 and 2 cell development: dependency on antigen dose and differential toll-like receptor ligation. J Exp Med 197, 101-109 (2003).

49. Chen, Y.L., Chen, T.T., Pai, L.M., Wesoly, J., Bluyssen, H.A. \& Lee, C.K. A type I IFN-Flt3 ligand axis augments plasmacytoid dendritic cell development from common lymphoid progenitors. J. Exp. Med. 210, 2515-2522 (2013).

50. Relman, D.A., Domenighini, M., Tuomanen, E., Rappuoli, R. \& Falkow, S. Filamentous hemagglutinin of Bordetella pertussis: nucleotide sequence and crucial role in adherence. Proc. Natl Acad. Sci. USA 86, 2637-2641 (1989). 